\title{
Introducing HEP to schools through educational scenaria
}

\author{
C.Kourkoumelis ${ }^{1, a}$ and S.Vourakis ${ }^{1, b}$ \\ ${ }^{1}$ Physics Faculty, University of Athens, Panepistimioupoli, llissia 15771, Greece
}

\begin{abstract}
Recent activities, towards the goal of introducing High Energy Physics in the school class, are reviewed. The most efficient method is a half or a full day workshop where the students are introduced to one of the large LHC experiments, follow a "virtual visit" to the experiment's Control Room and perform an interactive analysis of real data. Science cafes and visits to the CERN expositions are also very helpful, provided that the tours/discussions are led by an active scientist and/or a trained teacher. Several EU outreach projects provide databases rich with education scenaria and data analysis tools ready to be used by the teachers in order to bridge the gap between modern research and technology and school education.
\end{abstract}

\section{Introduction}

For many years the research centers had very little connection with the schools or with society in general. The information about cutting-edge research and technology has not been widely diffused and the school curricula in physics were based on basic principles, which were known for centuries. During recent years, the European Union (EU) has realized the existence of the gap between research and school education and launched many outreach programs in science which actively promote inquiry learning science education. In addition, the researchers in the most modern fields of science -who have been rather isolated and busy in their laboratories- have realized that they have an obligation to share their excitement and discoveries with students, teachers and the general public. However, despite the considerable investments made at the national and EU level in order to introduce innovative learning techniques in science education, teaching remains mostly lectue/blackboard centered. The students are told about science and asked to memorize facts instead of being taught to think scientifically and be engaged in playful episodes of learning.

In the field of High Energy Physics (HEP) recent events like the completion of the most powerful accelerator in the world, the LHC, and the subsequent discovery of the Higgs boson have attracted significant media attention and have generated great public interest. This often raised questions from the students which could not be answered by their teachers and an interest from both to know more and be actively involved has surfaced. The authors of this article have been very actively involved in this process by participating in a number of EU outreach projects and authoring and disseminating an event analysis tool for the ATLAS experiment's events, called HY.P.A.T.I.A (HYbrid Pupil's Analysis Tool for Interactions in ATLAS) [1]. Students using HYPATIA and other tools are interactively involved

\footnotetext{
ae-mail: hkourkou@phys.uoa.gr

be-mail: s.vourakis@gmail.com
} 
in HEP research and learn through hands-on experience. This helps HEP researches to disseminate the results of their studies as well as the latest technological developments which accompany them. In addition, the students learn about the latest results through inquiry, namely by asking questions and looking for answers. This gives students an insight into the "big science" research performed at CERN and stimulates an enthusiastic interest in it and at possible scientific careers.

\section{Tools/Activities for Introducing HEP to schools}

There is a variety of ways which can serve the goal of introducing HEP to schools. Starting from the traditional lectures, one can also use more focused hands-on experience. The CERN exhibitions as well as science cafes and the use of multimedia such as videos etc, help to achieve the goal. After about a decade of experimenting with different ways, we have found that the most effective one is a combination of lectures, tours to the specific experiments (ATLAS, CMS, AMS, Icecube) and handson experience. This combination which has been pioneered by the "Discover the COSMOS" EU project in the form of the so called "mini masterclass" - half day workshops- which can take place locally at the interested schools. The students are guided either remotely (by video-conferencing) or by a few visiting scientists and with the simultaneous help from their trained teachers, to perform the interactive HEP measurements. In addition, at the end of each workshop a few schools, at different locations but performing the same measurements, can be connected by video conference to compare and discuss their results. Usually these mini masterclasses are accompanied by a "virtual visit" to the experiment's control room [2], the center where the data were taken. This way the students have the opportunity to see the scientists at work "doing their shifts" and discuss with them in a long question and answer session, after the presentation of the experiment. In almost all of the above mentioned masterclasses, the presented experiment was the ATLAS experiment at the LHC, the virtual visits took place at the control room of ATLAS in Geneva and the HYPATIA event display was used for the interactive analysis of the data gathered by ATLAS. Several scenarios have been developed for the analysis which are presented in section 4.

During the period 2012-2014, the authors accompanied by members of the outreach consortia have visited over fifty schools in Greece from Thrace to Crete. In one occasion last March ten schools from the mainland plus two schools from Cyprus were simultaneously connected to the ATLAS Control Room (see Figure 1).

Remote mini masterclasses also took place mainly at CERN where visiting schools were guided remotely by researchers in Athens but with the active involvement of their teachers. Figure 2 shows a map of the recent remote mini masterclasses performed in Europe.

More lengthy masterclasses of a full day duration were organized by IPPOG [3]. According to the format of the International Masterclasses (IMC), each year about 10,000 high school students from all over the world go to one of about 160 nearby universities or research centers for one day in order to unravel the mysteries of particle physics by analyzing real data. During the IMCs the students were offered the opportunity to analyse data for all four main LHC experiments, according to the expertise of the tutors. Figure 3 shows Greek students at the University of Athens working on the ATLAS data during last year's IMC. In Greece in all IMCs organized every year (three in the Athens region, one in Thessaloniki and one in Crete), the students analyse data from ATLAS using the HYPATIA tool in the so called $\mathrm{Z}$ path [4] exercise. They use real events from the ATLAS detector to identify $\mathrm{Z}, J / \psi, \Upsilon$ and Higgs particles through the following decays: $\mathrm{Z} \rightarrow 21, J / \psi \rightarrow 21, \Upsilon \rightarrow 21, \mathrm{H} \rightarrow 41$ and $\mathrm{H} \rightarrow \gamma \gamma$. For this purpose the students combine the decay products in order to calculate the invariant mass of the parent particle.

During the period 2011-2012, the CERN mini expo visited Greece with stops to six different cities (Athens, Heraklion, Patras, Alexandroupolis, Kavala and Volos). For this tour the exhibition 


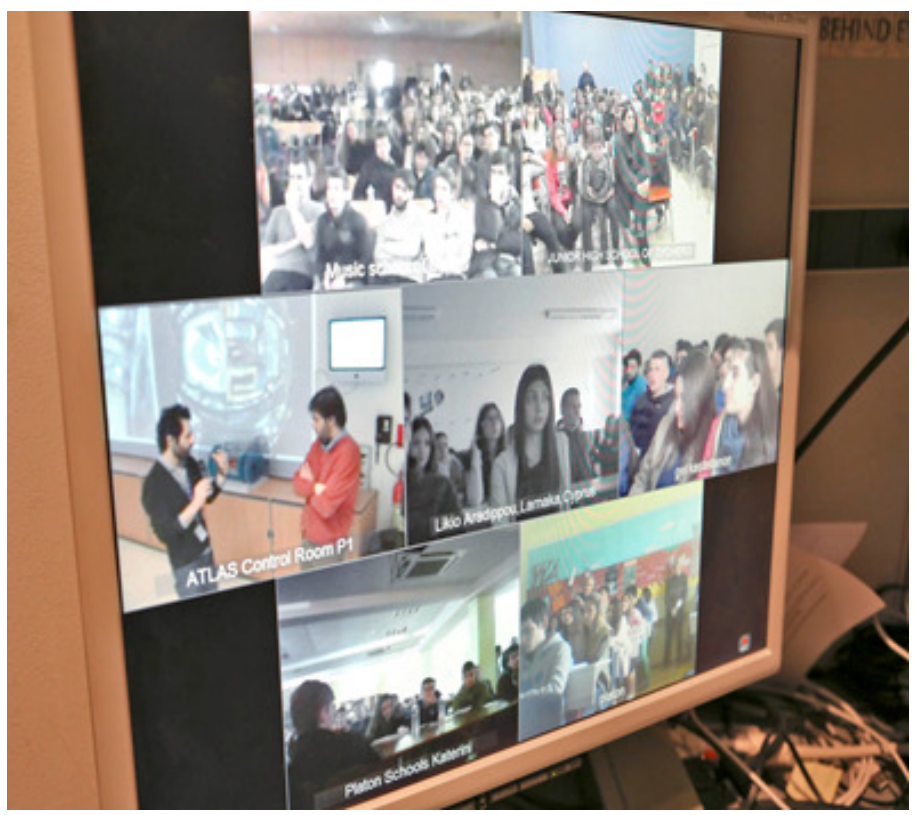

Figure 1. Ten Greek schools and two from Cyprus connected simultaneously with the ATLAS Control Room

was been upgraded with new material. The exhibition was combined with lectures and other activities -related to CERN- taking place at the hosting cities. The tour was a resounding success with more than 14,000 students visiting the exhibition and more than 650 teachers trained in the workshops that were conducted in parallel to the exhibition.

In the spring of 2014, the CERN "Accelerating Science" exposition (Figure 4) came to Athens for a month. It was hosted at the premises of the Eugenideio Foundation (science museum and planetarium) [5]. The exhibition was a huge success with more than 11,000 visitors, among them 4,000 students from 92 schools. Also about 120 teachers were trained in the use of HYPATIA in three workshops held in parallel to the exhibition.

Also during the 2013 CERN Open Days [6], a large crowd of about 3,000 visitors came to the ATLAS stand and had hands-on experience with the ATLAS event analysis tools (HYPATIA, Minerva, Collider) as well with the CERN outreach material for younger ages (LHCgame and CERNland).

\section{Resources/funding available}

The tools mentioned in the previous section were developed in the framework of the ATLAS outreach activities and by several EU projects. The EU projects which have already ended are "Learning with ATLAS@CERN" (LLP-1-2008-1) [7], "Pathway to Inquiry based science education" (SiS-20102.2,1-1) [8] and "Discover the COSMOS" (FP7-INFRASTRUCTURES-2111-2) [9]. The approach that the "Discover the COSMOS" implemented was to bring the use of "cutting-edge" e-Science applications to school students. The "Discover the COSMOS" activities introduced students to concepts and ideas of "big science" in the fields of Astronomy and HEP. The project activities such as the 


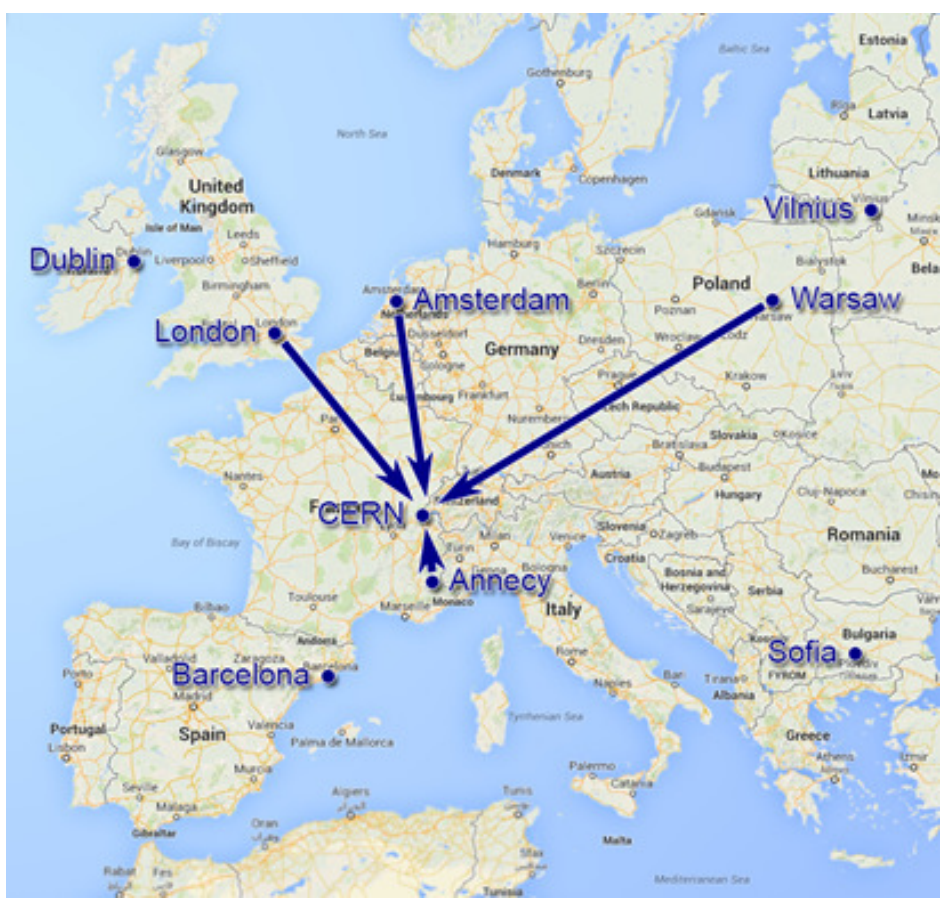

Figure 2. Mini masterclasses in Europe

analysis tools for real events collected by the ATLAS and CMS experiments at CERN and the remote real-time access to a number of telescopes were available to the students locally at their schools (mini and e-Masterclasses). The project designed and promoted to a network of teachers and students, a large number of learning scenaria and educational activities which are available on the project's portal (Figure 5). The portal ensures the sustainability of the project since it is available beyond the lifetime of the project with its technical infrastructure is linked with the Open Discovery Space eLearning platform [10] that aims to be the central European educational content aggregator in the next two years.

The new on-going EU projects which promote the implementation of HEP in the classroom are "Go-Lab" and "Inspiring Science education". The "Go-Lab" FP7-ICT-2011-8 [11] European collaborative project started in November 2012 and has a four year duration. Its goal is to create a unique federation of online, remote laboratories and data sets in all branches of science, based on the principles of inquiry learning and learning spaces structured in a pedagogical and scaffolded way. The project is addressed to a) students of the age of seven to eighteen who can perform personalized experiments b) teachers who are supported with scenaria for best practices and c) lab owners, since it provides wider dissipation and implementation of their labs. During the initial phase of the project about 48 labs have already been integrated to its repository following structured Inquiry Learning Spaces (ILSs). Four of them which use the HYPATIA tool are described in the next section. 


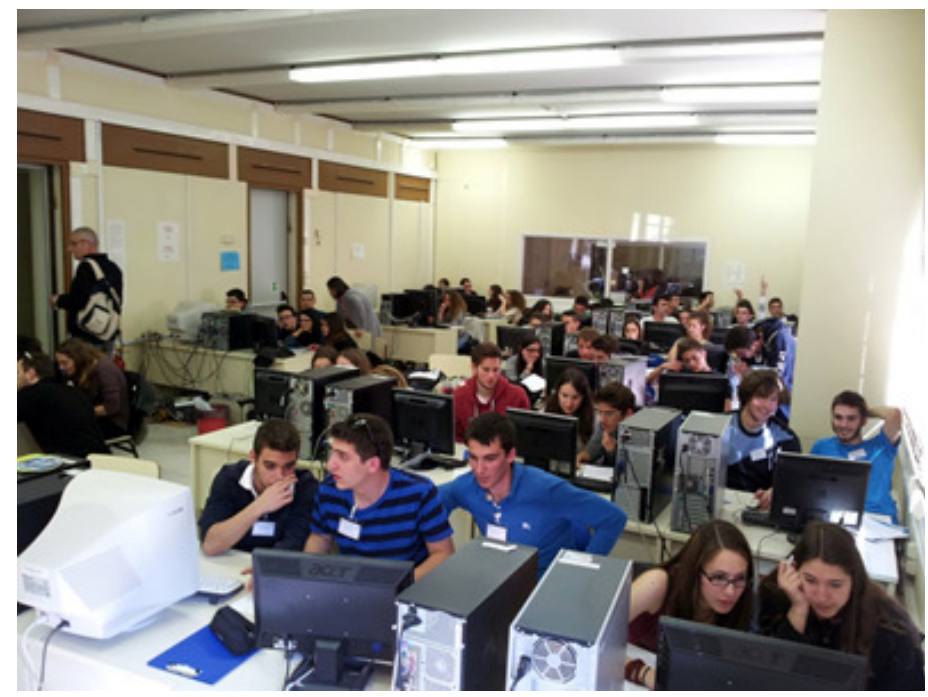

Figure 3. Students attending the 2014 IMC at the University of Athens
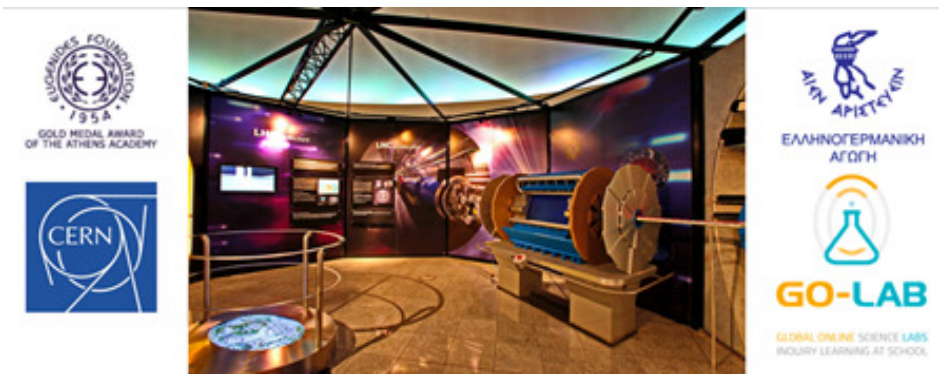

Figure 4. CERN Expo in Athens

The "Inspiring Science Education" CIP-ICT-PSP2012.23 project [12] began in April 2013 and it will run for 40 months. It aims at contributing to the implementation of the "Digital Agenda for Europe" by contributing to the modernization of education and training in sciences. This will be achieved while being in line with the recommendations of the Rocard Report [13], that sets the basics for the introduction of the Inquiry Based Approach in the science curricula of the Member States. The project implements large-scale pilots in Europe and beyond. The aim is to reach 5,000 primary and secondary schools in 15 European Counties during its lifetime.

All five of the above mentioned EU projects gave the opportunity to the members of the corresponding outreach teams to build "professionally" structured educational scenaria for the implementation of HEP in schools by profiting from the collaboration with science educators. In addition the 


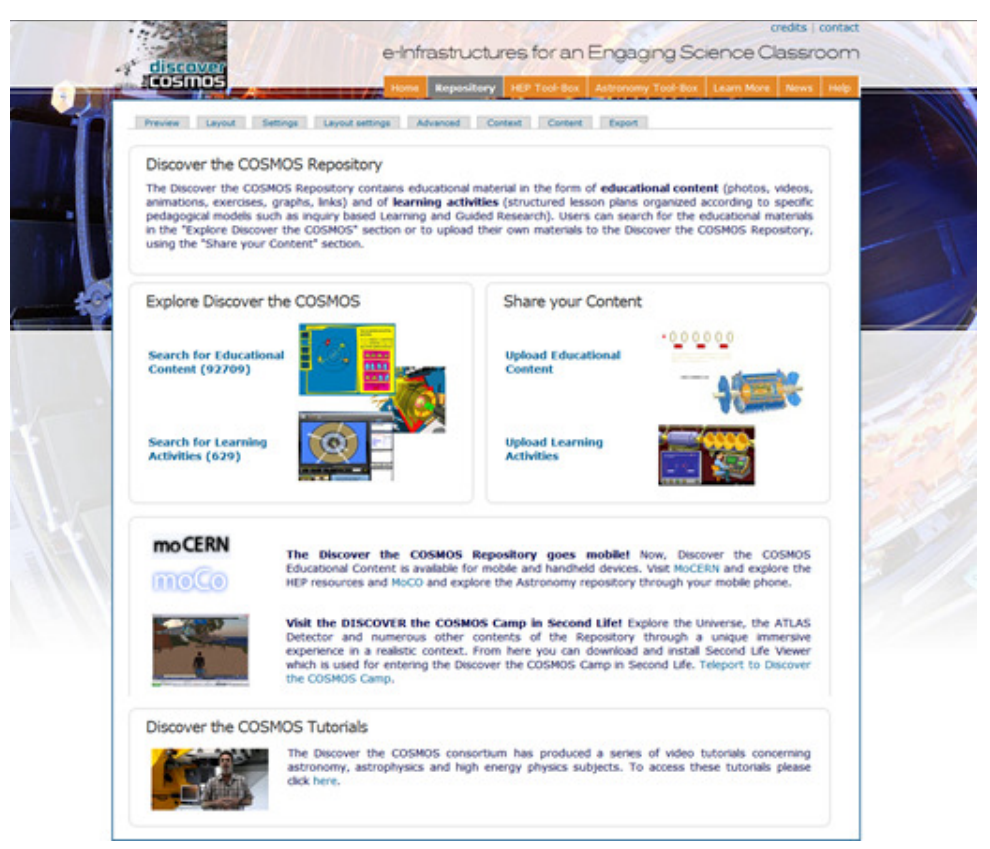

Figure 5. The "Discover the COSMOS" repository

projects provided support for reaching a large number of schools, research centers and universities by actually visiting them and providing "online" information, instructions and help.

\section{Educational scenaria with the use of HYPATIA}

HYPATIA offers a graphical representation of the products of proton collisions registered by the ATLAS detector. Students using the event display can interact with the events and in this way study the fundamental building blocks of nature and their interactions. At the same time, they learn how the ATLAS gigantic state-of-the-art detector works.

HYPATIA has been developed at the University of Athens since 2007, in cooperation with the Belgrade Institute of Physics during the first years (2007-2010). The full (offline) version of HYPATIA has been used in the International Physics Masterclasses since 2009. It uses java and is therefore compatible with most operating systems and it is freely available at hypatia.phys.uoa.gr along with event files, instructions and supporting material.

In addition to the offline version, since 2010 the University of Athens has developed an online version of HYPATIA which is intended to be used locally in schools without the need of installation of additional software. Recently it has been implemented as a web application which is supported by all OSs. It is available at hypatia.iasa.gr in Greek, English, German and French and includes various event files for the exercises described below, as well as instructions, help, exercise descriptions and solutions.

In the framework of the "Go-Lab" project four exercises have been developed recently and can be found in the Go-Lab portal in the form of ILSs which follow the full path of inquiry learning namely: 
orientation, conceptualization, investigation, conclusion and discussion. Detailed step-by-step instructions are given for all four exercises (educational scenaria) which are the following: Discover the $\mathrm{Z}$ boson, Hunt of the Higgs boson, Conservation of momentum and Measurement of the Magnetic field using the giant ATLAS detector.

\subsection{Discover the $\mathrm{Z}$ boson}

In this exercise [14] the students study the leptonic decays of the $\mathrm{Z}$ bosons to an electron-positron or muon-antimuon pair. In the first part, they are given detailed instructions and exemplary event files to become familiar with the identification of electrons and muons, using the corresponding length of the tracks and the signatures they leave in the calorimeters and the muon detectors. Then the students learn how to calculate the invariant mass of the parent particle from its decay products and how to check if the decays products satisfy some criteria like high $p_{T}$, isolation etc. As the last step, they produce histograms of the invariant masses and study the values separately for electron-positron and muon-antimuon as well as the corresponding widths. They can also compare the $\mathrm{Z}$ mass value with the one from the bibliography and discuss deviations.

\subsection{Hunt for the Higgs boson}

The Higgs exercise [15] builds upon the experience gained during the previous exercise, which is prerequisite. The students look for the production of two $\mathrm{Z}$ bosons in order to discover the Higgs boson through its decay to two $\mathrm{Z}$ bosons, followed by the decay into four leptons $(\mathrm{H} \rightarrow 4 \mathrm{l})$. The four leptons can be pairs of either electron-positron or muon-antimuon. In order to make the hunt simpler, the events contained in the online "Higgs" event file, are events which contain two on-shell Z bosons, produced by overlapping two real $\mathrm{Z}$ events. As a consequence, the mass of the few Higgs events contained in the file is not the mass of the discovered Higgs boson $(125 \mathrm{GeV})$ but close to $2 m_{Z}$.

\subsection{Conservation of momentum}

This exercise [16] is aimed at younger students and is directly related to the school curriculum. Its aim is to demonstrate that the same fundamental physics principle which applies to the macroscopic objects (e.g. two colliding spheres), applies to the world of the most fundamental particles which are the constituents of matter.

The principle of the exercise is shown in Figure 6. The left canvas is a slice of the ATLAS detector perpendicular to the colliding beams visualized by the HYPATIA online version. In this view the momentum before collision is zero (since the beams initially have only longitudinal momentum) and should be zero after the collision as well, if momentum is conserved. The students add the transverse momenta of all tracks which appear in the table below the canvas (red circle). The vector which has a magnitude equal to the vector sum but opposite in direction, is the missing transverse momentum. The students discuss reasons for being non zero (if it is so) and also compare their results with the $E_{\text {Tmiss }}$ calculated by the analysis tool; its magnitude is shown surrounded by the blue circle and its direction is shown by the red dotted line.

In this exercise we use specially selected events with very few tracks (muons, which are easily identifiable) so that the students can easily add them up (blue insert of Figure 6). 


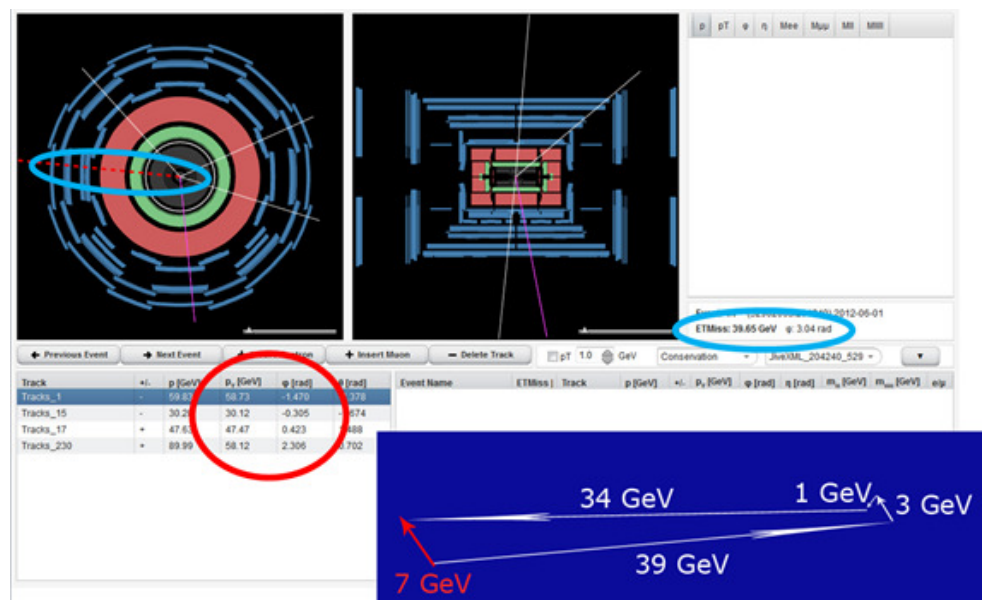

Figure 6. Conservation of momentum in the ATLAS detector

\subsection{Magnetic field measurement}

This is the second exercise [17] that is directly related to the school curricula. It is a direct application of the Lorenz force which is exerted on charged particles moving in a magnetic field. The ATLAS detector comprises of several huge magnets which bend the charged particles in order to measure their momenta (see Figure 7). The cylinder shown in green is a solenoidal magnet of uniform field which surrounds the collisions' point. The tracks get bent by the solenoid field which is parallel to the beams

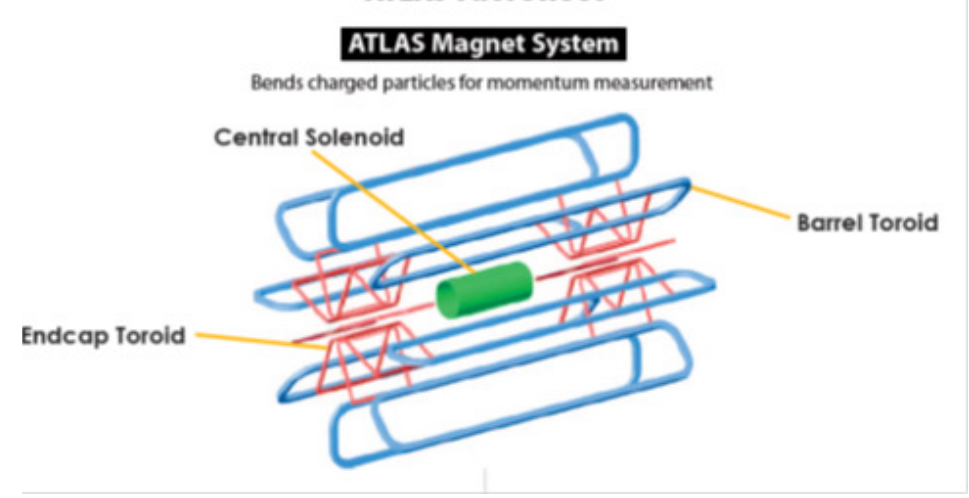

Figure 7. Schematic representation of the ATLAS magnets

(z-axis). Since the field lines are parallel to beams, the Lorentz force

$$
F=q *(v \times B)
$$


bends the charged particles in the plane perpendicular to the beams. This is shown in the left canvas of figure 8 . The force exerted on this plane is the centripetal force which is equal to the Lorenz force:

$$
\left(m * v_{T}^{2}\right) / R=q * v_{T} * B \Rightarrow p_{T} / R=q * B
$$

The particles' trajectories are circles and if their $p_{T}$ is between $500-700 \mathrm{MeV} / \mathrm{c}$, then the diameter of the circles is contained within the $2.4 \mathrm{~m}$ diameter of the solenoid. For simplicity only these tracks appear in the specific event file. The students select a track out of the event file; its $p_{T}$ is known since

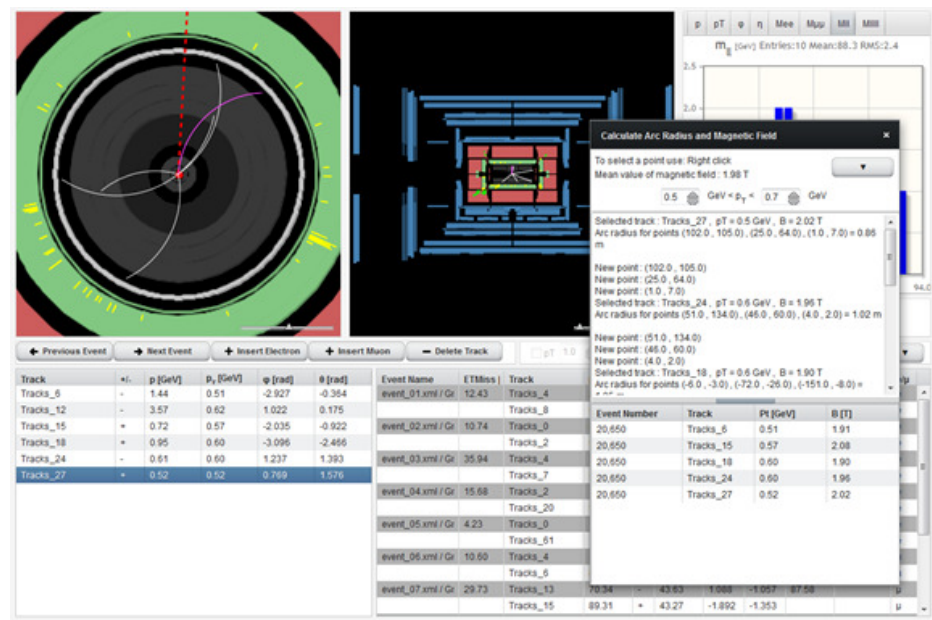

Figure 8. Screenshot of HYPATIA for B measurement

the corresponding track is highlighted in the track momenta table. The radius of the bent track $\mathrm{R}$ (arc radius) is calculated by HYPATIA by right clicking on three different well separated points on the track (by fitting a circle to that track). Knowing $\mathrm{R}$ (in $\mathrm{m}$ ) and $p_{T}$ (in $\mathrm{GeV} / \mathrm{c}$ ), the magnitude of the field strength (B in Tesla), can be calculated using equation (3).

$$
p_{T}(G e V / c)=0.3 * R(m) * B(T)
$$

The students can estimate B for different tracks and different events, take its average value, study its agreement to the nominal value of the B field strength of the ATLAS solenoid (see right insert of Figure 8). The teachers can also launch a discussion of statistical errors and deviations in measurements.

\section{Conclusions}

During the past few years a great effort has been made to exploit the recent publicity about CERN and the discovery of the Higgs boson in order to introduce the cutting-edge research and technology to high schools. To this end, several EU outreach projects have greatly helped by organizing masterclasses, mini-masterclasses and by enriching their repositories with educational scenaria- ready to be used by teachers- and best practices directly related to HEP. The "Discover the COSMOS" project alone, has reached 5,700 teachers and about 31,000 Students. 
During the European Strategy meeting in Brussels in May 2013, where the CERN Council updated the European Strategy for particle physics at the European Commission meeting, "Discover the COSMOS" and "Go-Lab" were one of the ten "talking" points presented as "on-line labs" to the CERN council, ministers and EU officials. The Strategy Report brochure distributed [18], stated under the chapter "society and skills": "The Discover the COSMOS and the Go-Lab projects are new ways of bringing frontier science to schools".

The two CERN exhibitions have helped thousands of people, among them hundreds of students and teachers to get acquainted with CERN and HEP. In Greece, this is shown by the significant increase in the number of Greek students and schools visiting CERN during the past two years (figures 9,10$)$.

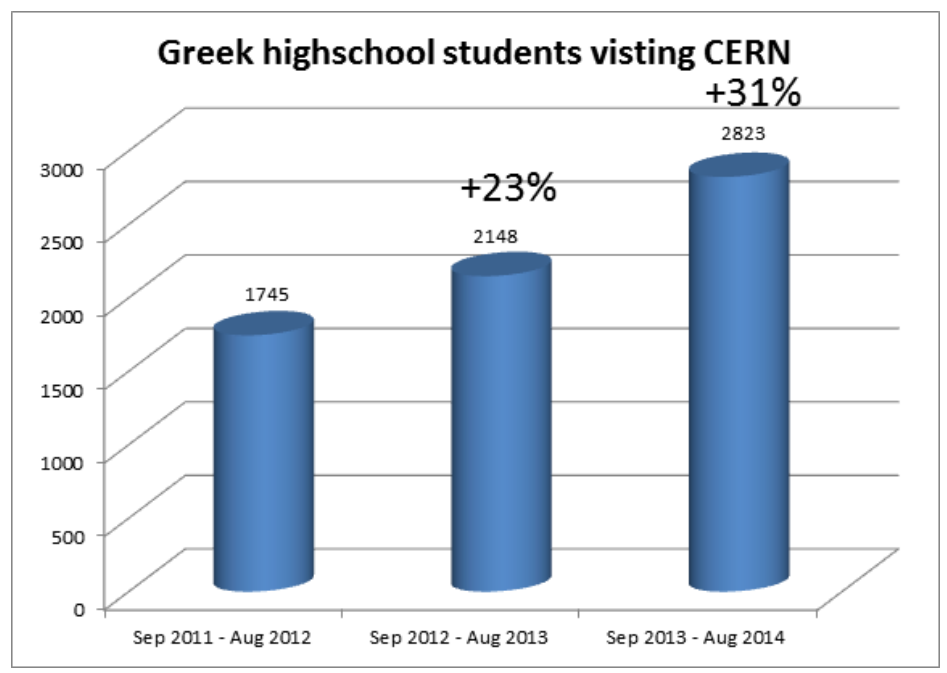

Figure 9. Increase in the number of Greek high school students visiting CERN during the last two years

Another example of the impact that the above actions have had on Greek students and teachers is the fact that the "Odysseus' Comrades" team from Varvakios Pilot School in Athens has won the "Win a Beam line" competition [19] of CERN. About three hundred teams submitted proposals to the competition; the Greek team together with a Dutch team won and will set up their experiments at CERN in September. The teacher who led the team, A. Valadakis, is an active and enthusiastic follower of our outreach team. He had his students participate in many of our masterclasses and has been trained in the use of HYPATIA. He has also taken his students to visit CERN several times. This is a great example of the impact of such actions extending beyond the classroom and the given educational scenaria. Under the right circumstances, students and teachers can profit from the knowledge transfered to them and possibly construct their own exercises or experiments.

\section{Acknowledgments}

This research has been co-financed by the European Union (European Social Fund - ESF) and Greek national funds through the Operational Program "Education and Lifelong Learning" of the National 


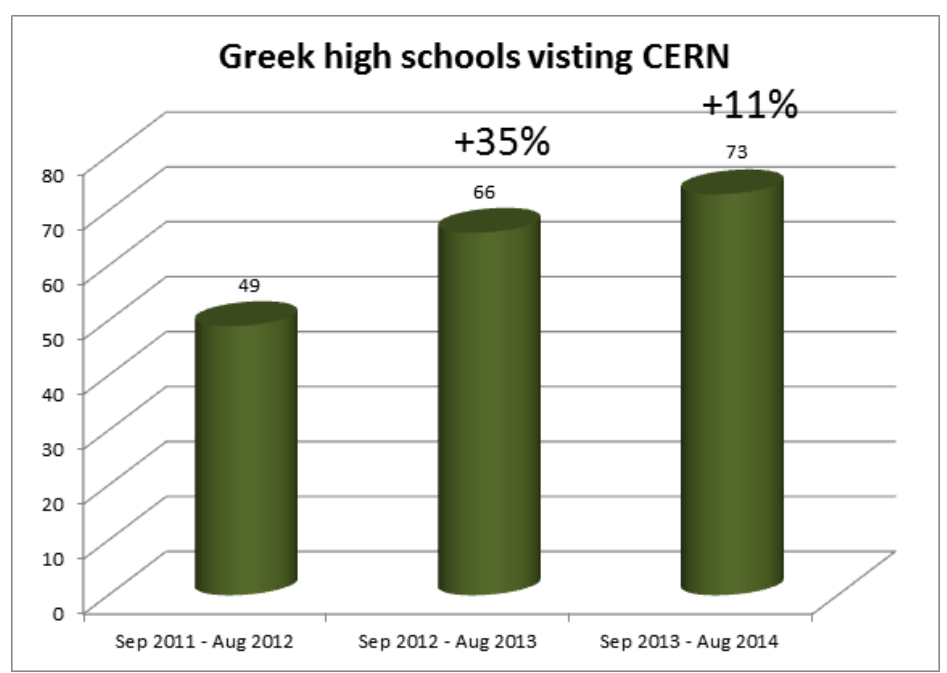

Figure 10. Number of Greek schools visiting CERN during the last three years

Strategic Reference Framework (NSRF) - Research Funding Program: THALES. Investing in knowledge society through the European Social Fund.

\section{References}

[1] C. Kourkoumelis and S. Vourakis, "HYPATIA-an online tool for ATLAS event visualization" , Phys. Educ. 49, 21 (2014).

[2] http://atlas-live-virtual-visit.web.cern.ch/atlas-live-virtual-visit

[3] http://ippog.web.cern.ch

[4] http://atlas.physicsmasterclasses.org/en/zpath.htm

[5] http://www.eugenfound.edu.gr

[6] http://opendays2013.web.cern.ch

[7] http://www.learningwithATLAS-portal.eu

[8] http://www.pathway-project.eu

[9] http://www.portal.discoverthecosmos.eu 
[10] htrp://www.opendiscoveryspace.eu

[11] http://www.go-lab-project.eu/frontpage

[12] http://inspiring-science-education.net

[13] Rocard M et al.Science Education NOW:EC high Level Group on Science ISBN 978-92-7905659-8:s.n.,2007

[14] https://graasp.epfl.ch/\#item=space9249

[15] https://graasp.epfl.ch/\#item=space9276

[16] https://graasp.epfl.ch/\#item=space7569

[17] https://graasp.epfl.ch/\#item=space9354

[18] http://cds.cern.ch/record/1551933/files/Strategy_Report_LR.pdf

[19] http://home.web.cern.ch/students-educators/updates/2013/11/schools-competition-win-beamline 